\title{
Sondeo ecológico rápido de las comunidades de peces tropicales en un área de explotación minera en Costa Rica
}

\section{Mario Espinoza Mendiola}

Centro de Investigación en Ciencias del Mar y Limnología. Escuela de Biología, Universidad de Costa Rica, San Pedro de Montes de Oca, 2060 San José Costa Rica; marioespinozamen@yahoo.com

\author{
Recibido 06-VII-2007. Corregido 26-V-2008. Aceptado 06-IX-2008.
}

\begin{abstract}
Rapid ecological assessment of tropical fish communities in a gold mine area of Costa Rica. Gold mining impacts have generated a great concern regarding aquatic systems and habitat fragmentation. Anthropogenic disturbances on the structure and heterogeneity of a system can have an important effect on aquatic community stability. Ecological rapid assessments (1996, 2002, and 2007) were employed to determine the structure, composition and distribution of tropical fish communities in several rivers and smaller creeks from a gold mining area in Cerro Crucitas, Costa Rica. In addition, species composition and relative abundance were related with habitat structure. A total of 35 species were registered, among which sardine Astyanax aeneus (Characidae) and livebearer Alfaro cultratus (Poeciliidae) were the most abundant fish (71\%). The highest species richness was observed in Caño Crucitas $(s=19)$ and Minas Creek $(s=18)$. Significant differences in fish communities structure and composition from Infiernillo river and Minas creek were observed $(\lambda=0.0$, $\left.\mathrm{F}_{132,66}=2.24, \mathrm{p}<0.001\right)$. Presence and/or absence of certain species such as Dormitor gobiomorus, Rhamdia nicaraguensis, Parachromis loiseillei and Atractosteus tropicus explained most of the spatial variation among sites. Habitat structure also contributed to explain differences among sites $\left(\lambda=0.004, \mathrm{~F}_{60,183}=5.52, \mathrm{p}<0.001\right)$. Substratum (soft and hard bottom types) and habitat attributes (elevation, width and depth) explained most of the variability observed in Infiernillo River, Caño Crucitas and Tamagá Creek. In addition, a significant association between fish species and habitat structure was observed. This study reveals a high complexity in tropical fish communities that inhabit a gold mine area. Furthermore,it highlights the importance of habitat heterogeneity in fish community dynamics. The loss and degradation of aquatic systems in Cerro Crucitas can have a strong negative effect on fish community structure and composition of local species. A better understanding of the use of specific habitats that serve as essential fish habitats can improve tropical fish conservation and management strategies, thus increasing local diversity, and thereby, the biological importance of the area. Rev. Biol. Trop. 56 (4): 1971-1990. Epub 2008 December 12.
\end{abstract}

Key words: Tropical fish communities, anthropogenic disturbance, gold mine, Costa Rica, ecological rapid assessment, species composition.

El impacto antropogénico de actividades mineras ha generado una gran preocupación por el deterioro de los hábitats acuáticos y la fragmentación de los sistemas naturales (Castilhos et al. 1998, Akçay y Moon 2004, Meck et al. 2006). Uno de los aspectos ecológicos fundamentales, es propiamente el impacto, directo e indirecto, de la actividad minera sobre las redes hidrográficas (e.g. ríos, quebradas, humedales y lagunas naturales). De acuerdo a Lake (2000), las perturbaciones que afectan los sistemas naturales son el resultado de dos eventos principales: 1) el disturbio mismo (producto de la aplicación de diferentes procesos capaces de alterar la estabilidad del sistema), y 2) la respuesta de los organismos ante dicho disturbio. Este disturbio puede producirse como un pulso, una presión constante, o una presión en aumento, y es preciso cuantificar sus efectos, para así poder evaluar las medidas necesarias capaces de mitigar cualquier riesgo existente (Zwane et al. 2006). 
En 1993 se inicio la exploración y prospección geológica, para la explotación aurífera en el Cerro Crucitas, región norte de Costa Rica. El proyecto consiste de tres actividades principales: 1) la extracción mecánica en etapas, mediante celdas de minado de la saprolita, 2) la licuefacción y transporte del mineral, y 3) el tratamiento en una planta de refinería con carbón en pulpa. La actividad de extracción de oro ha sido pospuesta en varias oportunidades por las autoridades ambientales nacionales, y aún en el 2007, permanece a la espera de la aprobación de estudios de impacto ambiental (E. Bermúdez comm. pers.). Aunque el diseño del proyecto contempla medidas que pretenden mitigar cualquier amenaza sobre el ambiente, es necesario considerar los escenarios posibles, capaces de alterar el equilibrio del sistema, y que puedan influir en el estado de las comunidades acuáticas que habitan las cuencas y micro-cuencas, próximas al área del proyecto. Rojas (2002), resume estos impactos, tomando en cuenta tres aspectos fundamentales del desarrollo de minerías de oro en el pasado: 1) la alteración del caudal principal y los niveles del flujo de agua (Ngcobo 2006), 2) la descarga de sedimentos terrígenos derivados de procesos de construcción y extracción del mineral (SolísPorras 1993, Wood 1997, Venter y VanVuren 1997), y 3) el uso de productos químicos con algún grado de toxicidad ambiental para el tratamiento de la saprolita (Porcella et al. 1997, Castilhos et al. 1998, Malm 1998).

La combinación de estos disturbios puede tener efectos negativos en todas las escalas de organización biológica, desde daños en la estructura celular, hasta respuestas a nivel de ecosistema (Peplow y Edmonds 2005). No solo se vería afectado el estado y condición de los organismos acuáticos, sino también la estructura, composición y distribución de las comunidades acuáticas. La ictiofauna de la zona norte de Costa Rica ha sido ampliamente estudiada en diversos trabajos taxonómicos (Bussing 1976, Koenig et al. 1976, Konings 1989, Bussing 1998), sin embargo el conocimiento de aspectos ecológicos específicos, como la organización y estructura de las comunidades de peces tropicales, relaciones ecológicas de las especies ante diversos procesos bióticos y abióticos, y las consecuencias del deterioro y fraccionamiento del hábitat, ha sido relativamente escaso (Burcham 1988, Bussing 1993, 1994). Ante al desarrollo de una minería de oro en el Cerro Crucitas, se han generado amplías descripciones ecológicas en los ríos y quebradas cercanas al área de impacto (McElligott y Slaney 1996, Rojas 2002), facilitando el diagnóstico de las comunidades acuáticas y mejorando el entendimiento del funcionamiento de las comunidades de peces en sistemas alterados (Freitas et al. 2005, Meck et al. 2006).

Varios estudios sugieren que la heterogeneidad estructural del hábitat, es uno de los componentes más importantes en moldear la estructura de las comunidades de peces tropicales, influyendo así en la distribución de especies y en patrones de segregación espacial en diversas escalas temporales (Ault y Johnson 1998, Espinoza y Salas 2005). Es de esperar que sistemas con mayor heterogeneidad espacial pueden proporcionar una mayor disponibilidad de micro-ambientes, favoreciendo a su vez, a una mayor riqueza y colonización de especies (Honran et al. 2000, Walter y Haynes 2006). Además, Lake (2000) señala que perturbaciones sobre la estructura y heterogeneidad de los hábitats acuáticos pueden influir de forma negativa en la estabilidad de las comunidades presentes.

El objetivo del estudio es generar una fuente de información de línea base, que permita evaluar los efectos inmediatos y cambios temporales, que puedan surgir con el desarrollo de una minería de oro en el Cerro Crucitas, Costa Rica. Para esto se emplearon sondeos ecológicos rápidos (1996, 2002, 2007), en los que se determinó la estructura, composición y distribución de peces tropicales que habitan los ríos y quebradas en el área de la minería. Además, se estimó la heterogeneidad espacial del hábitat, para determinar las variables que contribuyen a explicar cambios en la composición y estructura de las comunidades presentes. Finalmente, se analizó la relación entre la estructura del hábitat y las asociaciones de especies observadas. 


\section{MATERIALES Y MÉTODOS}

Área de estudio: El Cerro Crucitas está ubicado en la región norte de Costa Rica y consiste principalmente de lomas arcillosas con elevaciones menores a los 100 m.s.n.m, y zonas bajas que originalmente estaban cubiertas por bosque tropical primario (Fig. 1). El reciente impacto de actividades antropogénicas como la agricultura y ganadería ha producido cambios evidentes en el paisaje. Una gran porción de bosque primario ha sido deforestado, fragmentado o convertido en pastizal (McElligott y Slaney 1996). Existen cuatro redes hidrográficas principales: los ríos Tirisias e Infiernillo, y los Caños Crucitas y Venada, los cuales drenan al Norte con el río San Juan (Fig. 1). Sin embargo, únicamente el río Infiernillo y el Caño Crucitas se encuentran en el área próxima a la minería. El río Infiernillo posee un área total de $1500 \mathrm{Km}^{2}$ y una longitud aproximada de $50 \mathrm{Km}$. Este río atraviesa por bosques intervenidos, con pastizales para pastoreo, además de bosques secundarios y zonas reforestadas. La Cuenca Crucitas posee un área de $26 \mathrm{Km}^{2}$ y una longitud aproximada de $10.50 \mathrm{Km}$. Esta cuenca se caracteriza por bosques moderadamente densos, con algunos pastizales y zonas reforestadas (Rojas 2002).

\section{Metodología de sondeo ecológico rápido} (SER): Los cambios en la estructura y composición de las comunidades de peces tropicales fueron evaluados mediante sondeos ecológicos rápidos (SER), realizados durante JunioJulio 1996 (SER1), Noviembre 2002 (SER2) y Febrero-Marzo del 2007 (SER3), de acuerdo a la metodología empleada en McElligott y Slaney (1996) y Rojas (2002). Cada SER fue realizado como un sondeo ecológico independiente, en donde se documentó la ictiofauna presente en el área de la minería, además de anotaciones ecológicas. Debido a diferencias en métodos de captura (ver sección recolección de especimenes) e incongruencias en la selección de sitios de muestreo, la mayor parte de los

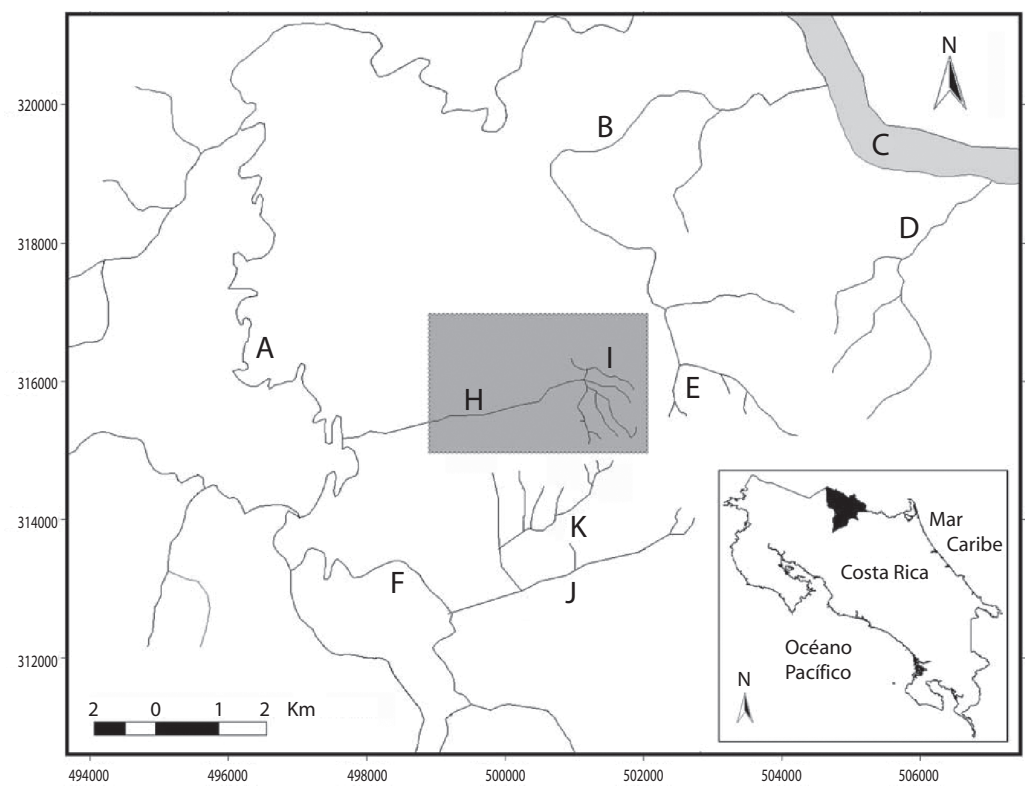

Fig. 1. Área de estudio. Cerro Crucitas, región norte de Costa Rica (rectángulo sombreada delimita la zona principal de extracción de oro del proyecto minero. Principales ríos y quebradas: A - Río Infiernillo, B - Caño Crucitas, C - Río San Juan, D - Caño Venada, E - Quebrada Descubrimiento, F - Río Chamorro, G - Quebrada Llano Verde, H - Quebrada Minas, I - Quebrada Tamagá, J - Quebrada Zaballo y K - Quebrada Cascada. 
análisis estadísticos empleados en el presente estudio se restringieron al SER3. No obstante, Rojas (2002) intento seleccionar sitios similares a los de McElligott y Slaney (1996), para establecer así, una base de comparación temporal. En el presente estudio, se recopiló toda la información existente, seleccionando estaciones similares que permitieran analizar patrones espaciales de las comunidades de peces tropicales, principalmente usando el SER2 como base temporal. Además, los resultados del presente estudio pretenden comparar y discutir observaciones integrales del SER1 y SER2 que puedan ser pertinentes para la conservación y manejo de peces tropicales en ambientes alterados. En el SER1 se monitorearon 9 ríos y quebradas (15 estaciones), en el SER2 se evaluaron 7 ríos y quebradas (10 estaciones), y en SER3 7 ríos y quebradas (28 estaciones), que incluían principalmente, las cuencas del Caño Crucitas y Río Infiernillo, además de varias quebradas y lagunas de menor extensión (Cuadro 1). El número de estaciones se refiere a diferentes localidades del mismo sitio (río o quebrada) en donde se realizaron muestreos. Durante los años 2004-2006, la extensión del área del proyecto minero fue reducida considerablemente. Algunas estaciones incluidas en los SER 1 y 2 fueron omitidas del SER3, y se incluyeron otras que presentaban un mayor riesgo de impacto directo sobre los ecosistemas acuáticos.

Otro aspecto importante que fue incluido específicamente en el SER3, fue la evaluación de la heterogeneidad espacial en la estructura y composición de las comunidades de peces tropicales en los sitios próximos a la minería. Las siguientes mediciones estructurales del hábitat fueron tomadas en segmentos similares del río durante cada muestreo: ancho (m), profundidad promedio $(\mathrm{m})$, altitud $(\mathrm{msnm})$, cobertura de vegetación ribereña, velocidad de corriente y sustrato (\% limo, $\%$ arena-grava, $\%$ hojarascatroncos, $\%$ piedras, $\%$ roca masiva). La vegetación ribereña ( 1 - bosque denso, 2 - bosque denso/alterado, 3 - bosque alterado/pastizal, 4 - bosque muy alterado), y velocidad de corriente (1 - nula, 2 - nula a baja, 3 - baja a moderada,
4 - moderada a alta, y 5 - alta/rápidos) fueron estimadas de acuerdo a una escala cualitativa de valores, y posteriormente estandarizadas como porcentaje de cobertura e intensidad de corriente, respectivamente. Finalmente, se incluyó información bibliográfica de los hábitos alimenticios de las especies observadas durante los SER (1-2-3) para elucidar posibles relaciones tróficas (Bussing 1998).

Recolección de especímenes: Se emplearon redes fijas de enmalle en mono-filamentos $(15.2 \times 1 \mathrm{~m}$, apertura de malla: $25-75 \mathrm{~mm})$, redes de cerco manual $(4-8 \times 1 \mathrm{~m}$, apertura de malla: $5 \mathrm{~mm}$ ), red de mano circular (30 $\mathrm{cm}$ diámetro, $40 \mathrm{~cm}$ profundidad), esparavel en polifilamentos (1.4 m diámetro, apertura de malla: $5 \mathrm{~mm}$ ) y trampas manuales (nasas). Además, durante el SER1 se utilizó un equipo de electropesca portátil a $300 \mathrm{~V}$ y $50 \mathrm{~A}$ (modelo Smith Root 15A) (para mas detalle ver McElligott y Slaney 1996). Para los análisis estadísticos y relaciones multivariadas se empleó, únicamente, información de las redes de cerco manual que tuvieran al menos 5 muestras por estación. Los individuos colectados fueron congelados luego de su captura, y posteriormente identificados mediante claves taxonómicas (Bussing 1998), en el laboratorio húmedo del Museo de Zoología de la Universidad de Costa Rica. Además, se tomó la longitud estándar (distancia entre la porción más extrema de la mandíbula superior y la base del pedúnculo caudal), longitud total (distancia entre la porción más extrema de la mandíbula superior y el final de la aleta caudal) y el peso (g) de cada uno de los individuos colectados.

Análisis de datos: Los datos fueron estandarizados en abundancia relativa tomando en cuenta el esfuerzo de muestreo realizado en cada estación. Además se calculó el número total de especies (s), y la abundancia y riqueza promedio por estación. Para determinar si existían diferencias en la longitud de individuos entre sitios se realizó un análisis no paramétrico de Kruskal-Wallis. Los patrones de composición y distribución de especies 


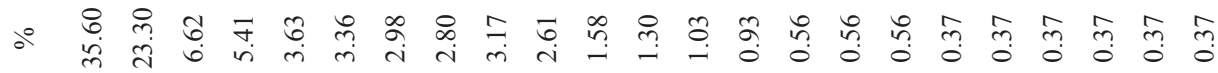

$$
\begin{aligned}
& \text { స్ّ } \\
& \text { 过 } \infty \quad n \text { in m }
\end{aligned}
$$

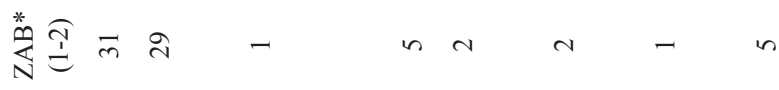

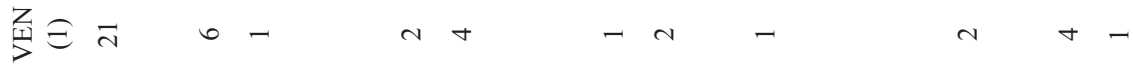

$$
\begin{aligned}
& \stackrel{\vartheta}{\ddots}
\end{aligned}
$$

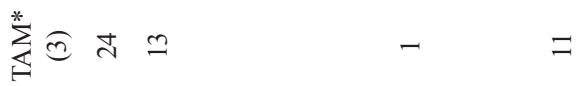

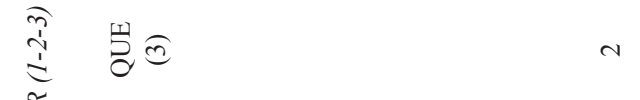

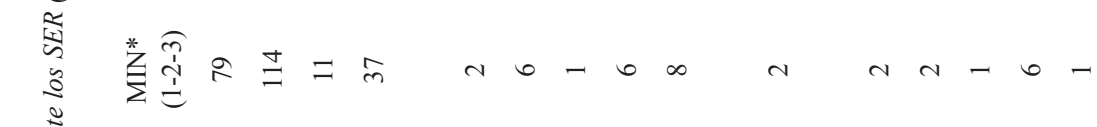

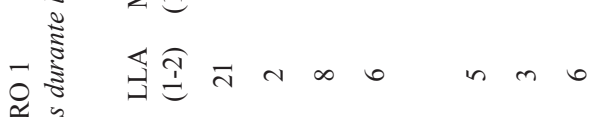

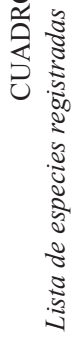

$$
\begin{aligned}
& \text { 莣 } \stackrel{\overparen{P}}{=} \sim \infty-\infty-\infty- \\
& \text { 중 }
\end{aligned}
$$

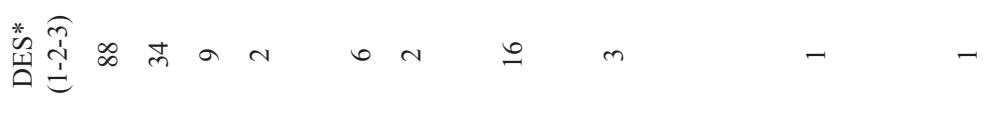

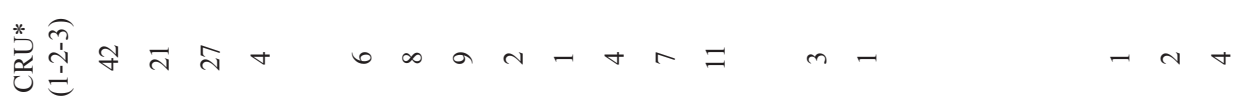

$$
\begin{aligned}
& \text { 岂こ के } \\
& \text { 芑 }
\end{aligned}
$$

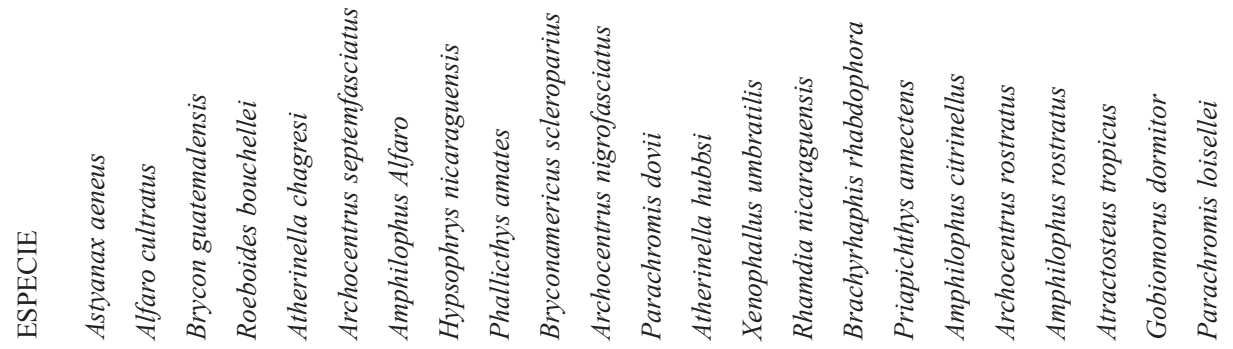




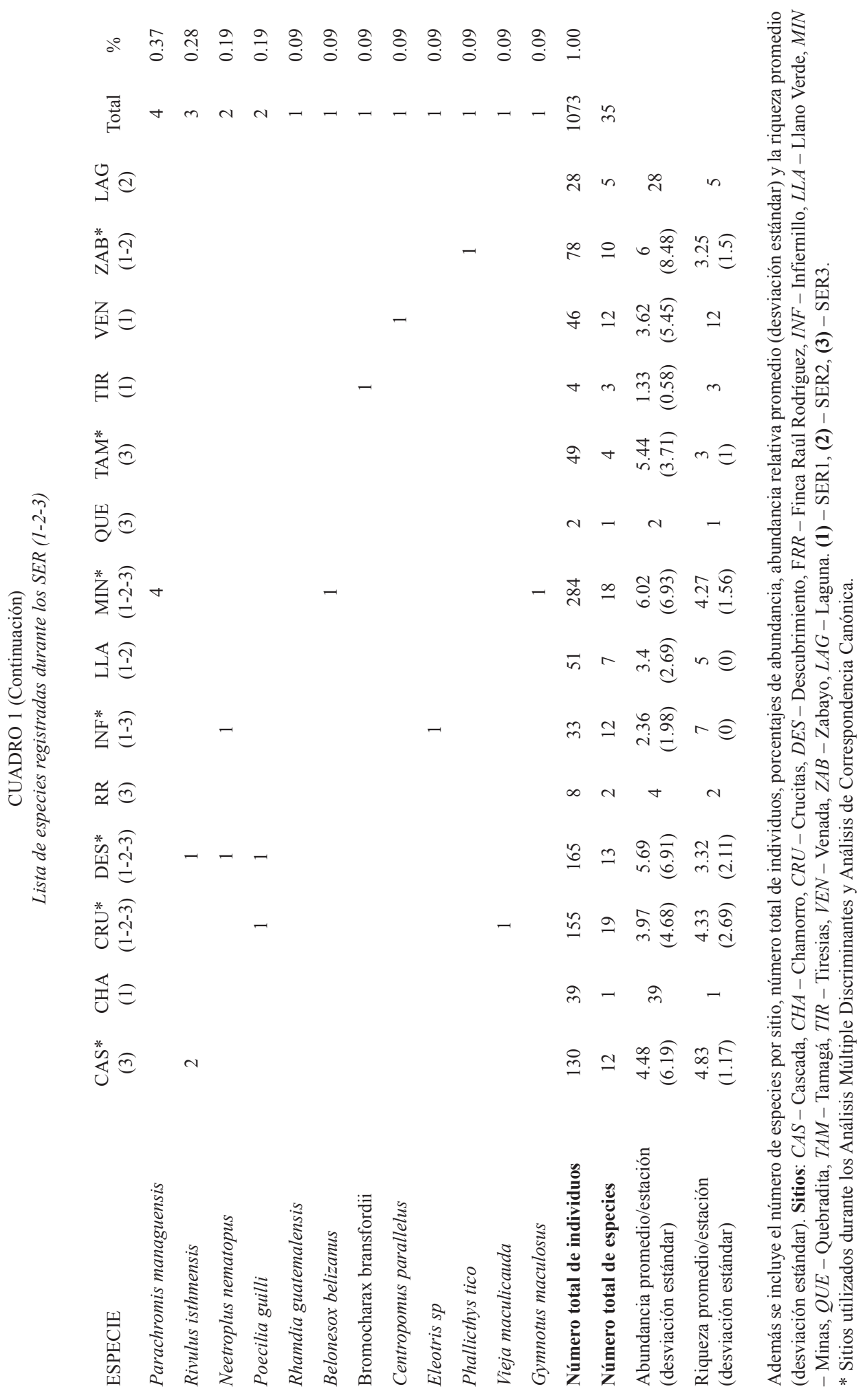


observadas se estudiaron mediante Análisis de Conglomerados por agrupamiento jerárquico, basado en el índice de similitud de Morisita como medida de clasificación y el método de unión por promedios (Krebs 1999, Quinn y Keough 2003). La variación espacial en la estructura y composición de las comunidades de peces fueron determinadas mediante Análisis Múltiple Discriminante (AMD). La abundancia y número de especies fueron utilizadas en el AMD para determinar cuales especies contribuyen a explicar la variabilidad espacial observada entre sitios (Digby y Kempton 1987, Gotelli y Ellison 2004). También se utilizó el AMD para analizar la variabilidad estructural del hábitat (tipos de sustrato y atributos del hábitat) y determinar las variables que explican las diferencias entre sitios. Finalmente, la relación entre la estructura del hábitat y las asociaciones de especies observadas, fueron evaluadas mediante Análisis de Correspondencia Canónica (ACC). Todos los análisis multivariados fueron realizados con el Programa Estadístico Brodgar 2.5.1. La abundancia de especies fue normalizada mediante la transformación $\sqrt{(x+1)}$, las variables del hábitat fueron transformadas mediante $\log _{10}(x+1)$ y los porcentajes mediante la transformación seno $^{-1} \sqrt{(x+1)}$ (Zar 1999).

\section{RESULTADOS}

Durante los SER (1-2-3) se registraron 35 especies, pertenecientes a 11 familias de peces tropicales, para un total de 1073 individuos (Cuadro 1). Las especies más comunes fueron la sardina plateada Astyanax aeneus (Characidae), la olomina Alfaro cultratus (Poeciliidae), la machaca Brycon guatemalensis (Characidae) y la sardina alma seca Romboides bouchellei (Characidae), las cuales fueron observadas en la mayoría de los sitios. Estas especies constituyen el $71 \%$ de la abundancia total observada, $\mathrm{y}$ en general, ambas familias (Characidae y Poecilidae) constituyen el $79 \%$ de los individuos registrados durante los SER. No obstante, la mayor riqueza de especies se concentró en la familia Cichlidae $(\mathrm{s}=11)$. Entre los ríos y quebradas estudiados, el Caño Crucitas ( $\mathrm{s}=19$ ) y la quebrada Minas $(\mathrm{s}=17)$ fueron los sitios con mayor número de especies, seguidos por la quebrada Descubrimiento, el río Infiernillo y la Cascada, respectivamente (Cuadro 1). La distribución de las sardinas Atherinella hubbsi y A. chagresi se restringió al río Chamorro y al Caño Crucitas, lo cual podría deberse a características específicas del tipo de hábitat en el que se desarrollan. Únicamente durante el SER1 se reportó el pez gaspar Atractosteus tropicus. Esta especie fue observada en los Caños Crucitas y Venada, así como en el río Infiernillo. Sin embargo, actualmente sus poblaciones se encuentran amenazadas debido a la pesca intensiva y fragmentación de los hábitats acuáticos en la zona norte de Costa Rica.

Estructura y composición de las comunidades: En el análisis de conglomerados se destacan dos grupos importantes. El primero incluye, únicamente al río Chamorro, mientras que en el segundo grupo se encuentran los demás sitios. El río Chamorro presentó la mayor distancia de similitud, debido a que únicamente se registró una especie, la sardina A. chagresi. Los demás sitios se agrupan según la similitud en la estructura y composición de sus comunidades (Fig. 2). En este segundo grupo, también se observaron dos agrupaciones importantes, la primera corresponde a la Finca Raúl Rodríguez y la Quebradita, las cuales comparten un bajo número de especies y abundancia relativa. Además, ambas quebradas poseen un caudal reducido, con fondos blandos de limo y arcilla. En la segunda agrupación, se observó una gran similitud de especies entre el Caño Crucitas y el río Infiernillo, la Cascada y Minas, y Llano Verde y Zabayo, respectivamente. En este grupo, el río Tirísias fue el sitio más lejano, debido a la presencia de la sardina Bramocharax bransfordii, la cual estuvo ausente en el resto de los sitios (Cuadro 1, Fig. 2).

La estructura y composición de las comunidades de peces también varió significativamente entre sitios $\left(\lambda=0.000, \mathrm{~F}_{132,66}=2.24\right.$, $\mathrm{p}<0.001)$. La mayor variabilidad espacial se observó en el río Infiernillo y en la quebrada 


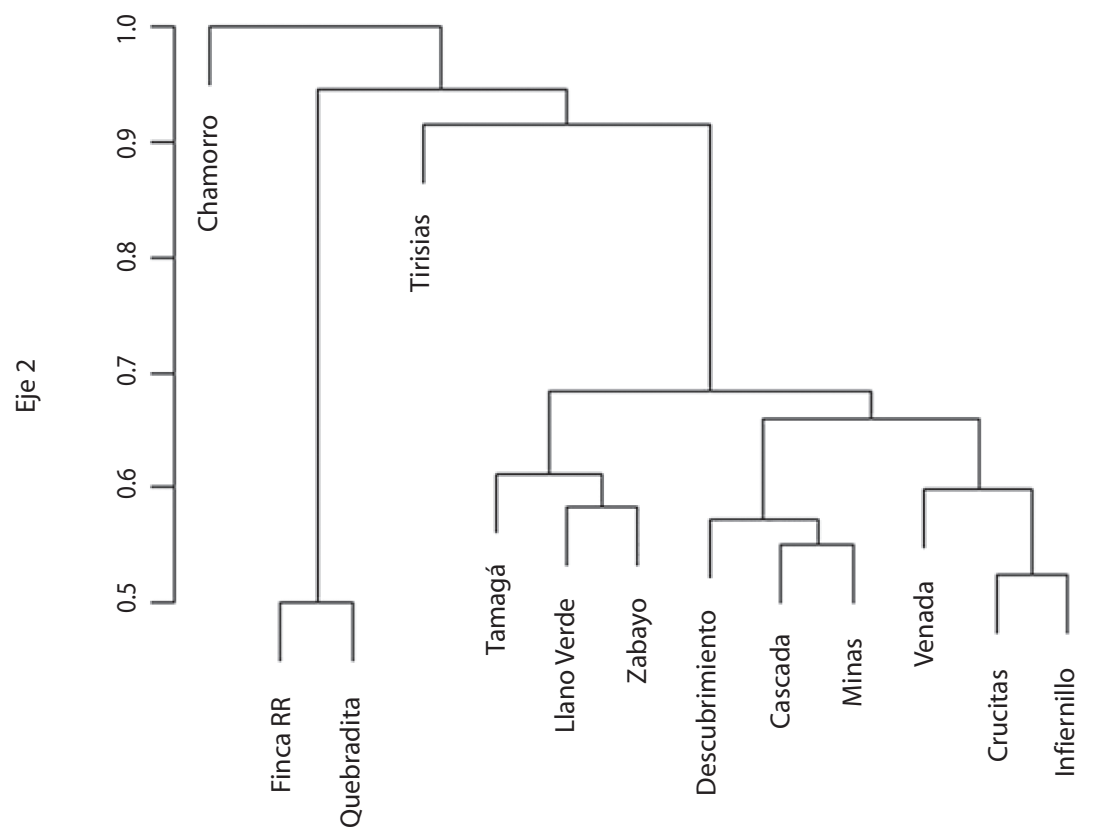

Eje 1

Fig. 2. Dendrograma de clasificación de los sitios (ríos/quebradas) evaluados durante los sondeos ecológicos rápidos (SER), mediante el análisis de conglomerados de las especies observadas. La medida de similitud empleada fue el índice de Morisita y se uso el método de aglomeración por promedios.

Minas. Además, se encontró una tendencia a la separación de Zabayo, Tamagá y el Caño Crucitas, del resto de los sitios (Fig. 3). De acuerdo al AMD los primeros tres ejes explicaron un $84 \%$ de la variabilidad ( 2 ejes explican el $74 \%$ ). En el primer eje, la guavina Gobiomorus dormitor, el barbudo Rhamdia nicaraguensis, el guapote Parachromis loiseillei, y el gaspar A. tropicus explicaron la mayor parte de la variabilidad observada entre sitios, mientras que en el segundo eje, la ausencia de la sardina A. hubbsi contribuye a explicar la variabilidad asociada al Caño Crucitas (Cuadro 2, Fig. 3).

Heterogeneidad estructural del hábitat: La heterogeneidad y estructura del hábitat también contribuyó a explicar las diferencias espaciales observadas (Cuadro 4, Fig. 4). Las dimensiones del hábitat, cobertura de vegetación ribereña y los tipos de sustrato variaron significativamente entre sitios $(\lambda=$ $\left.0.004, \mathrm{~F}_{60,183}=5.52, \mathrm{p}<0.001\right)$. De acuerdo al AMD, el río Infiernillo, el Caño Crucitas y la quebrada Tamagá presentaron la mayor variación espacial (Fig. 4). Los tres primeros ejes explicaron el 90\% (2 ejes explicaron el $68 \%$ ), siendo la elevación, el porcentaje de limo y roca masiva en el sustrato, las variables que contribuyen a explicar la mayor parte de la variación observada en el primer eje, mientras que en el segundo eje, el ancho del caudal contribuyo a explicar el resto de la variabilidad observada (Cuadro 3). Al estudiar la relación del ensamblaje de especies y la estructura del hábitat, se encontró que existen patrones de agrupación importantes (Fig. 5). La sardina A. hubbsi, el guapote Parachromis dovii y la mojarra Archocentrus nigrofasciatus 


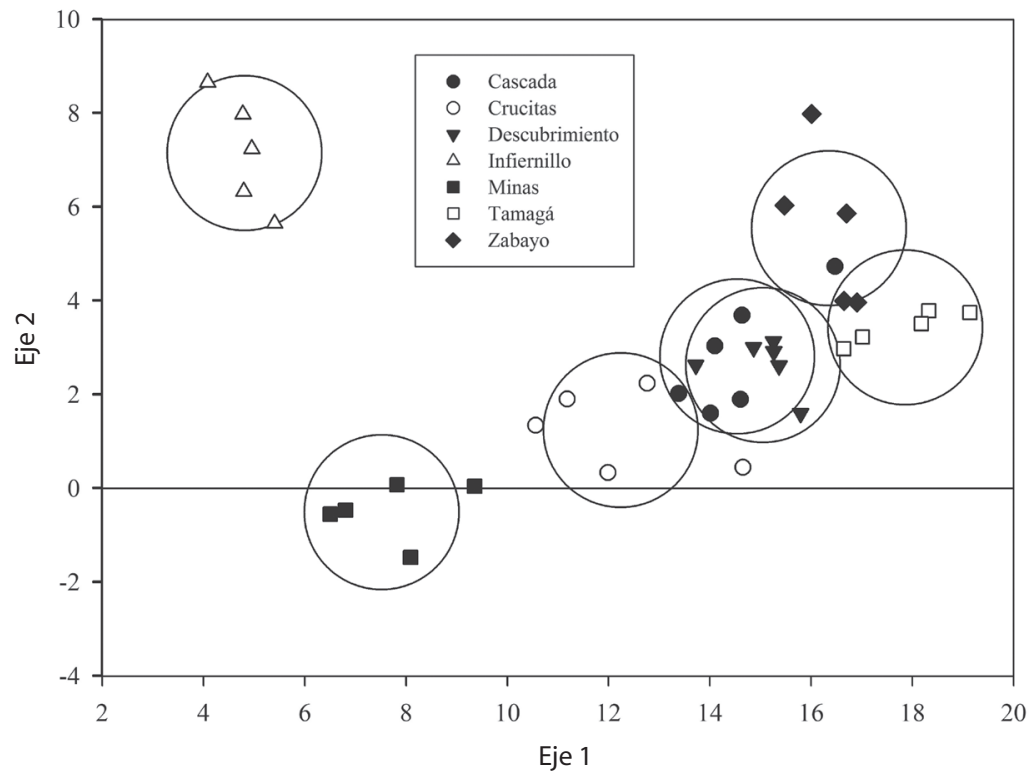

Fig. 3. Promedio de las funciones discriminantes del AMD. Ejes representan el número y abundancia de especies que explican la variabilidad entre sitios (ríos/quebradas) estudiado durante el SER2 y SER3. Círculos representan el promedio de la función discriminante $(95 \%$ área de traslape). $\$ Cascada, $\bullet$ Minas, $\square$ Crucitas, $\bigcirc$ Tamagá, $\Delta$ Descubrimiento, $\square$ Zabayo, Infiernillo.

\section{CUADRO 2}

Contribución de las especies registradas en el Análisis Múltiple Discriminante (datos estandarizados entre varianzas)

Especie

Alfaro cultratus

Archocentrus nigrirostris

Amphilophus rostratus

Archocentrus septemfasciatus

Amphilophus alfari

Astyanax aeneus

Atherinella hubbsi

Atractosteus tropicus

Brachyrhaphis rhabdophora

Brycon guatemalensis

Bryconamericus scleroparius

Gobiomorus dormitor

Hypsophrys nicaraguensis

Xenophallus umbratilis

Parachromis dovii

Parachromis loiseillei

Parachromis managuensis

Phallicthys amates

Priapicthys annectens

Rhamdia nicaraguensis

Roeboiedes bouchelli
Función discriminante 1

$-1.21$

11.8

8.25

1.59

1.71

$-0.81$

$-11.68$

$-36.67$

$-16.8$

$-2.56$

6.3

40.03

$-19.53$

7.49

$-21.22$

36.88

2.96

$-5.36$

$-10.42$

40.22

$-8.21$
Función discriminante 2

$-0.79$

$-1.43$

1.61

$-0.6$

0.2

0.95

$-28.35$

0.36

$-3.92$

$-0.82$

1

$-13.72$

12.47

7.91

10.76

31.64

1.93

0.84

$-15.48$

3.49

$-4.75$ 


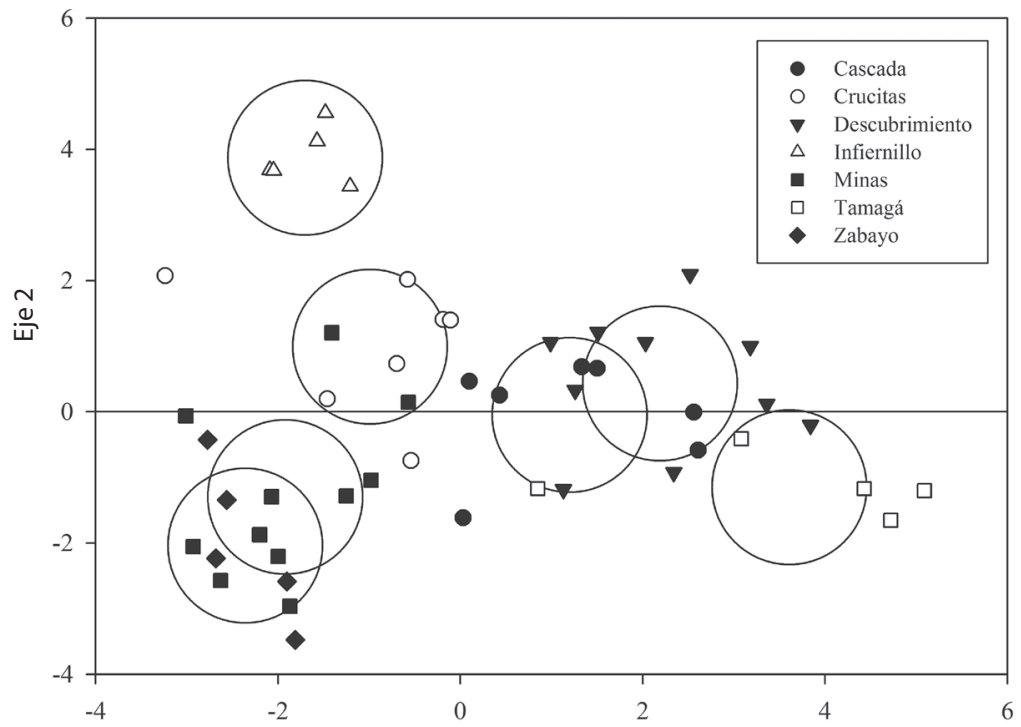

Eje 1

Fig. 4. Promedio de las funciones discriminantes del Análisis Múltiple Discriminante. Ejes representan la estructura del hábitat (atributos y tipos de sustrato) que explican la variabilidad entre sitios (ríos y quebradas) estudiados durante el SER 2 y SER3. Círculos representan el promedio de la función discriminante ( $95 \%$ área de traslape). $\diamond$ Cascada, $\bullet$ Minas, $\square$ Crucitas, Tamagá, $\triangle$ Descubrimiento, $\square$ Zabayo, $\bigcirc$ Infiernillo.

\section{CUADRO 3}

Contribución de los atributos del hábitat y tipos de sustrato en el Análisis Múltiple Discriminante (datos estandarizados entre varianzas)

\begin{tabular}{|c|c|c|c|}
\hline & Hábitat & Función discriminante 1 & Función discriminante 2 \\
\hline \multirow{4}{*}{ 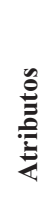 } & Altitud & 1.17 & 0.77 \\
\hline & Ancho & 0.56 & 2.07 \\
\hline & Profundidad & -0.38 & 0.11 \\
\hline & Velocidad & -0.3 & -0.1 \\
\hline \multirow{6}{*}{ 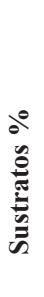 } & Vegetación rivereña & -0.11 & -0.2 \\
\hline & $\%$ Limo & -0.86 & -0.8 \\
\hline & $\%$ Arena/grava & -0.36 & 0.25 \\
\hline & $\%$ Vegetación/troncos & -0.36 & -0.6 \\
\hline & $\%$ Piedra & 0.57 & -0.3 \\
\hline & $\%$ Roca masiva & 0.8 & -0.4 \\
\hline
\end{tabular}




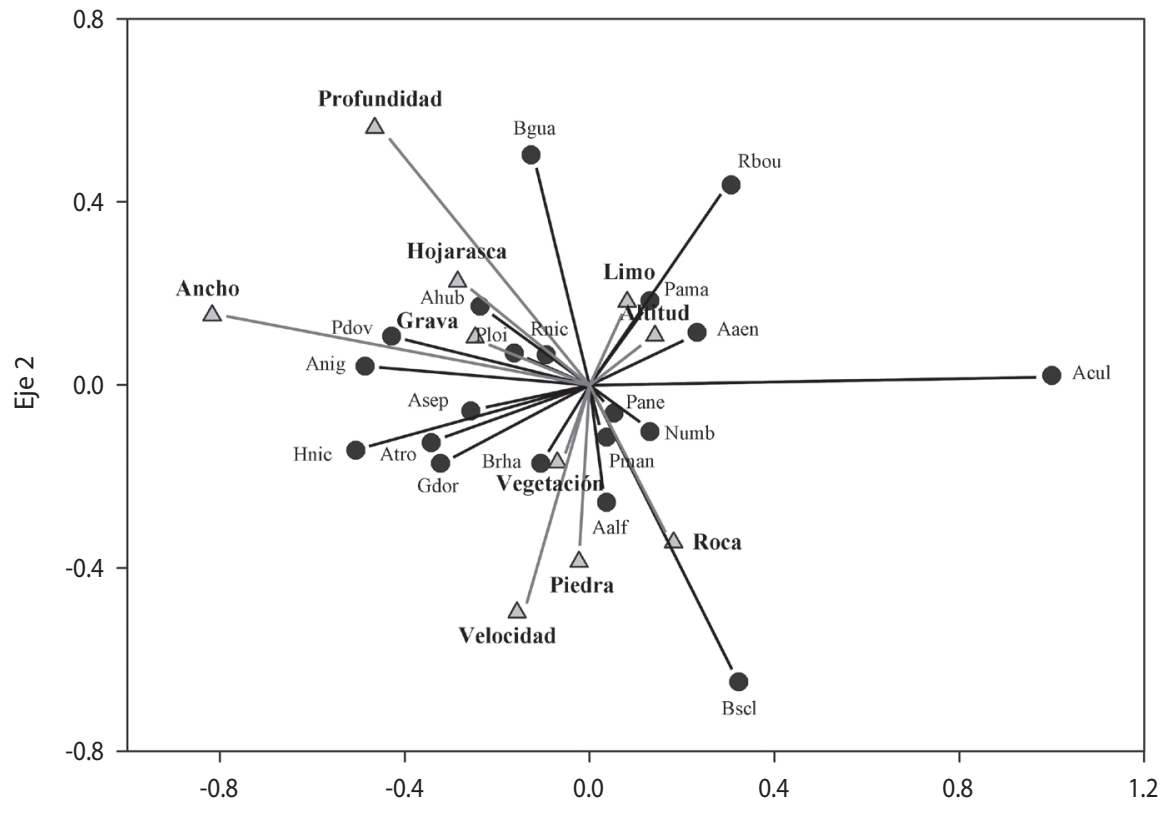

Eje 1

Fig. 5. Análisis de Correspondecia Canónica de relación entre la estructura del habitat y las asociaciones de especies observadas en los ríos y quebradas durante el SER3 (Prueba de Monte Carlo: $\mathrm{F}=2.17, \mathrm{p}=0.005,199$ permutaciones). Especies: Acul - Alfaro cultratus, Bscl- Brachyrhaphis scleroparius, Aalf - Amphilophus alfari, Numb - Xenophallus umbratilis, Pman - Parachromis managuensis, Pane - Priapicthys annectens, Brha - Brachyrhaphis rhabdophora, Gdor - Gobiomorus dormitor, Atro- Atractosteus tropicus, Hnic, Hypsophrys nicaraguensis, Pama - Phallicthys amates, Asep - Archocentrus septemfasciatus, Anig - Archocentrus nigrirostris, Pdov - Parachromis dovii, Ploi - Parachromis loisellei, Rnic - Rhamdia nicaraguensis, Ahub - Atherinella hubbsi, Bgua - Brycon guatemalensis, Rbou - Roeboides bouchelli y Aaen - Astyanax aeneus.

se asociaron con ríos y quebradas de mayor ancho y con un dominio de grava y vegetación en el sustrato. La machaca Brycon guatemalensis se observó, principalmente, en sitios de mayor profundidad. La sardina A. aeneus, olomina Phallicthys amates y la alma seca R. bouchelli se encontraron en sitios con fondos blandos de limo y arcilla. Finalmente, la sardina Bryconamericus scleroparius muestra una fuerte preferencia por ríos con mayor cobertura rocosa (Fig. 5). Los 3 primeros ejes del ACC explican un $40 \%$ de la variabilidad observada (Cuadro 5). La olomina A. cultratus fue la única especie que no parece mostrar ninguna relación con un hábitat en particular.
Estructura trófica: Al analizar los hábitos alimenticios de las especies registradas durante los SER se observó que existen dos grupos tróficos bien definidos: omnívoros e insectívoros. El grupo de omnívoros fue el más importante a lo largo de los SER (57\%; $\mathrm{s}=12$ ), y junto con el grupo de insectívoros, constituyen el $87 \%$ de la ictiofauna observada en el estudio (Fig. 6). El grupo de detritívoros y lepidófagos también fue representativo, con 6 especies y aproximadamente un $9 \%$ de la abundancia observada. A pesar de que el grupo de piscívoros fue poco abundante, se registraron 7 especies, lo cual podría tener un efecto importante sobre los patrones de distribución 
CUADRO 4

Promedio (DE) de los atributos del hábitat y tipos de sustrato registrado en cada uno de los sitios evaluados durante el SER3

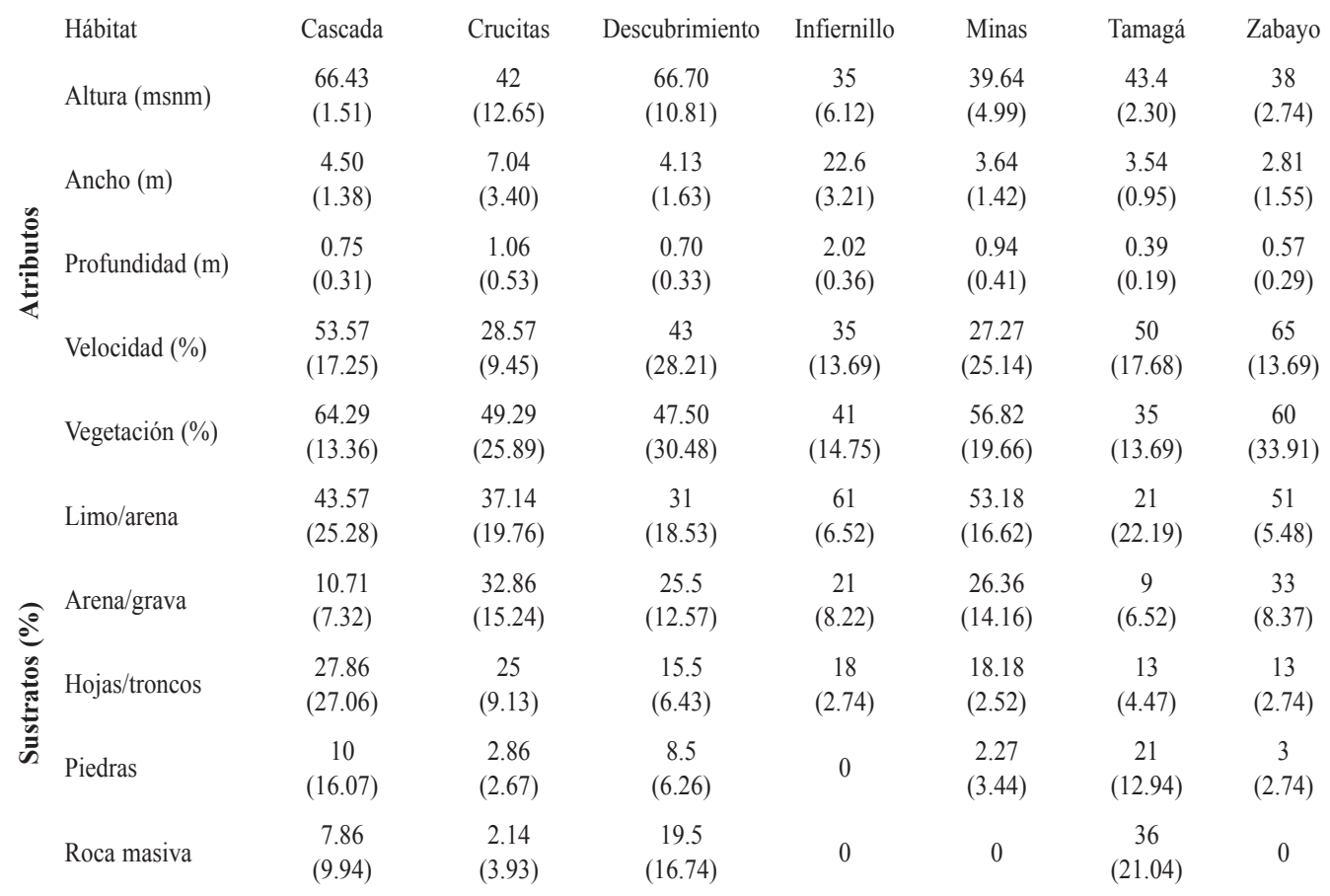

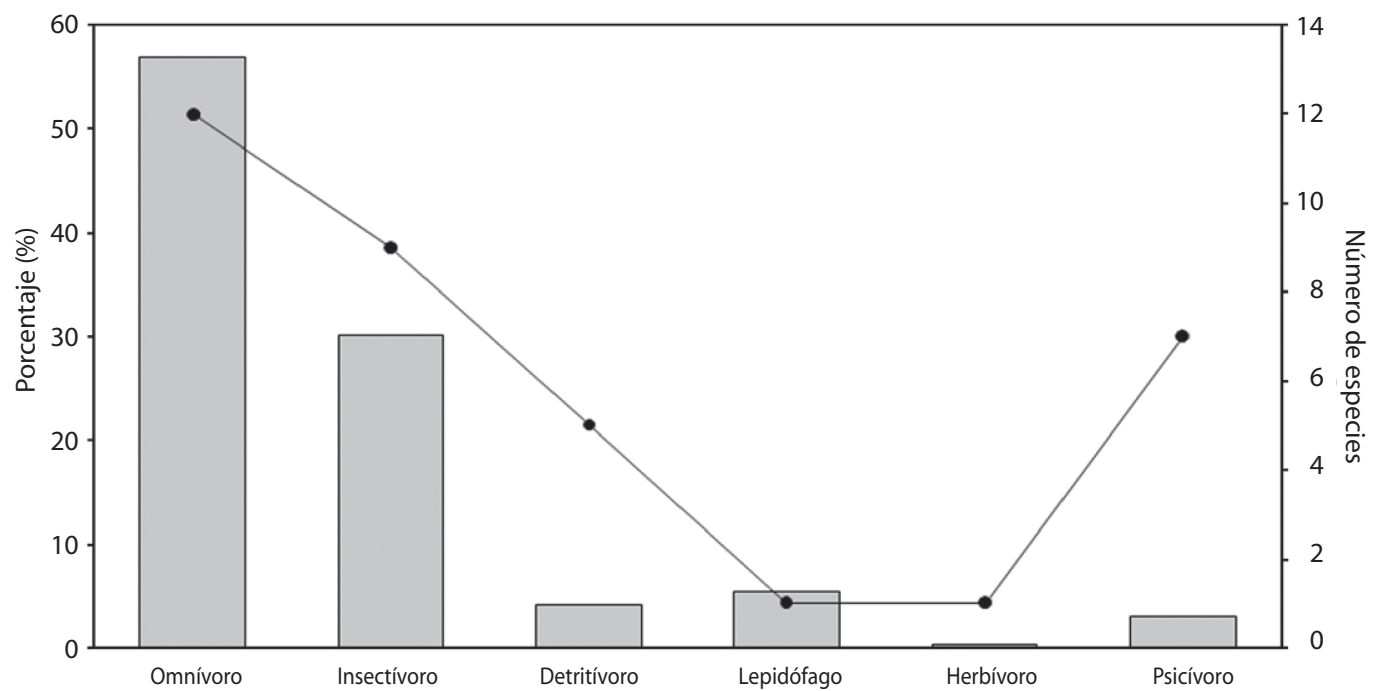

Fig. 6. Porcentaje de individuos y número de especies registradas durante los SER (1-2-3) según el grupo trófico Abundancia y - Número de especies). 
CUADRO 5

Porcentaje de masa, inercia y varianza explicada en el Análisis de Correspondencia Canónica de la relación entre las especies observadas y las variables de la estructura del hábitat (SER3)

$\begin{array}{lccc}\text { Especie } & \text { Masa (\%) } & \text { Inercia (\%) } & \text { Varianza } \\ \text { Alfaro cultratus } & 6.69 & 25.77 & 0.11 \\ \text { Bryconamericus scleroparius } & 4.71 & 9.06 & 0.06 \\ \text { Roeboides bouchelli } & 4.75 & 5.68 & 0.05 \\ \text { Hypsophrys nicaraguensis } & 4.38 & 4.27 & 0.04 \\ \text { Parachromis dovii } & 4.37 & 3.11 & 0.03 \\ \text { Astyanax aeneus } & 7.94 & 2.14 & 0.02 \\ \text { Gobiomorus dormitor } & 4.09 & 1.91 & 0.02 \\ \text { Atractosteus tropicus } & 4.06 & 1.89 & 0.02 \\ \text { Atherinella hubbsi } & 4.18 & 1.35 & 0.03 \\ \text { Archocentrus septemfasciatus } & 4.46 & 1.17 & 0.05 \\ \text { Amphilophus Alfaro } & 4.57 & 1.13 & 0.04 \\ \text { Astatheros rostratus } & 4.01 & 0.68 & 0.02 \\ \text { Brachyrhaphys rhabdophora } & 4.11 & 0.6 & 0.02 \\ \text { Parachromis loisellei } & 4.04 & 0.46 & 0.01 \\ \text { Neetroplus nematopus } & 4.19 & 0.43 & 0.03 \\ \text { Rhamdia nicaraguensis } & 4.11 & 0.2 & 0.01 \\ \text { Parachromis managuensis } & 4.01 & 0.19 & 0.01 \\ \text { Phallicthys amates } & 4.06 & 0.18 & 0.03 \\ \text { Priapicthys annectens } & 4.04 & 0.1 & 0.01\end{array}$

y segregación espacial de otras especies (Fig. 6). Además, se observó una gran variación en la longitud de los individuos capturados entre sitios (ámbito: 18-1000 mm LT) (Fig. 7). No obstante, únicamente los individuos del río Infiernillo (154.29 $\pm 207.17 \mathrm{~mm} \mathrm{LT})$ mostraron diferencias significativas (Kruskall-Wallis $\chi^{2}=$ 43.67, $\mathrm{gl}=6, \mathrm{p}<0.001)$.

\section{DISCUSIÓN}

Este estudio evidencia la complejidad en la estructura y composición de la las comunidades de peces tropicales que habitan las cuencas y micro-cuencas de la zona norte del país. Además, acentúa la importancia de la heterogeneidad estructural del hábitat como fuente potencial de micro-ambientes, que aumentan la riqueza de especies, inclusive a escalas locales (Ormonds y Roberts 1997, Ault y Johnson 1998). Rojas (2002) estimó que en el área de la minería de oro del Cerro Crucitas existen, aproximadamente, 35 especies de peces tropicales, lo cual constituye el $100 \%$ de las especies registradas durante los sondeos ecológicos rápidos (SER 1-2-3), y más del 55\% de las especies que han sido reportadas en los $9911 \mathrm{~km}^{2}$ de la Cuenca hidrográfica que incluye al Lago de Nicaragua-Río San Juan (PNUMA-OEA 1997, Bussing 1998). La sardina $A$. aeneus y la olomina $A$. cultratus fueron las especies más abundantes del estudio. De acuerdo a Bussing y López (1977), las sardinas en general (familia Characidae), son capaces de habitar regiones con mayor elevación y flujo del caudal, por lo que tienden a tener un mayor 


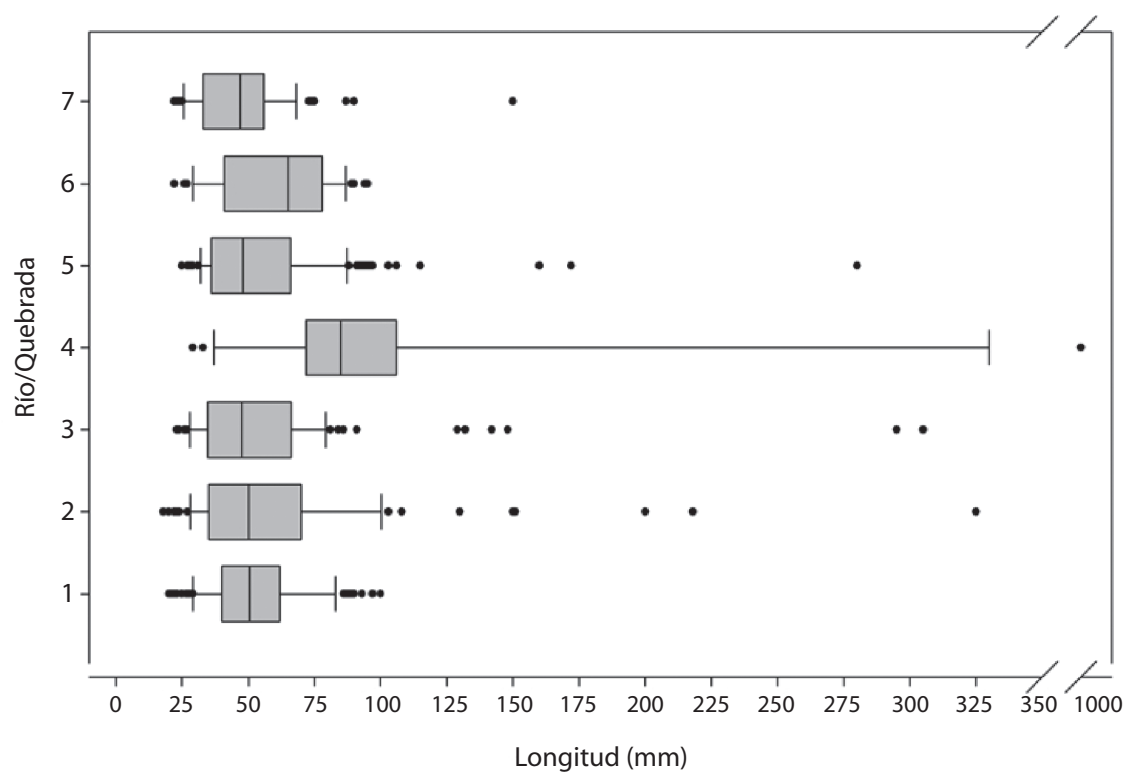

Fig. 7. Variación en la longitud total (desviación estándar) de individuos observados en los ríos/quebradas durante el SER3: 1 - Cascada, 2 - Caño Crucitas, 3 - Descubrimiento, 4 - Infiernillo, 5 - Minas, 6 - Tamagá y 7 - Zabayo. Barras grises incluyen la mediana, los percentiles 25, 50, 75 y 90, y las barras de error para cada sitio.

ámbito de distribución en los ríos y quebradas del país. A. aeneus se distribuye desde el nivel del mar hasta los 1000 m.s.n.m., mientras que A. cultratus está restringida a altitudes menores a los 300 m.s.n.m., principalmente en la vertiente Atlántica y región Norte del país (Bussing 1998).

Estructura y composición de las comunidades: Varias especies mostraron una fuerte evidencia de segregación espacial entre sitios. Los principales cambios en la composición de especies pueden explicarse por la ausencia y/o presencia de especies entre sitios. Mota y Cox (2003) señalan que los patrones de segregación espacial de especies pueden resultar de las diferencias en la estructura del hábitat. Otros estudios también aportan evidencia de que la riqueza y distribución de peces tropicales es inversamente proporcional a atributos del hábitat como la elevación y directamente proporcional al tamaño de la cuenca, ancho, profundidad, distancia del caudal principal (Guilliam et al. 1993, Honran et al. 2000, Rojas 2002). Esta situación es típica de muchos sistemas fluviales del mundo, siendo la altura uno de los factores más importantes (Lowe-McConnell 1987), pues esta inter-relacionado con la temperatura, el flujo y velocidad de corriente, y los niveles de saturación de oxígeno disuelto (Roldan 1992). A pesar de esta situación, el mayor número de especies se registró en el Caño Crucitas y en la quebrada Minas, y no en el río Infiernillo, el cual posee una mayor área, extensión y grado de complejidad estructural, lo cual podría favorecer a una mayor riqueza de especies. Sin embargo, la fuerte precipitación y el aumento en el caudal principal del río Infiernillo (SolísPorras 1993) limitan el acceso a las estaciones de muestreo y dificultan la captura de peces, lo cual podría estar subestimando el número de especies registradas.

La unión de la quebrada Minas con el río Infiernillo contribuye a explicar el alto número de especies y la similitud en la estructura y composición de sus comunidades. Freitas et al. (2005) encontraron que la mayor complejidad en la estructura de un río se encuentra 
a menores elevaciones, en la zona cercana a la desembocadura o en la unión con otros ríos y quebradas, en donde la heterogeneidad espacial y la abundancia de alimento proporcionan sitios ideales para una mayor riqueza de especies. Además, las dimensiones (ancho y profundidad) del caudal en ambos sitios, así como la variedad de sustratos, generan una alta heterogeneidad espacial, proporcionando una mayor abundancia de hábitats disponibles (Ibarra et al. 2005). Matthews (1986), señala que la adición continúa de especies, de riachuelos pequeños hacia grandes afluentes, es un patrón consistente en la mayoría de las comunidades de organismos acuáticos, tanto en zonas templadas como tropicales. La quebrada Minas podría estar "inyectando" especies hacia afluentes más grandes como el río Infiernillo. También es posible que algunas de las especies presentes en el río Infiernillo utilicen la quebrada Minas como zonas de crianza o refugios ante depredadores, durante etapas críticas del desarrollo.

Heterogeneidad estructural del hábitat: Al igual que la composición y distribución de especies varió espacialmente, también se observaron diferencias importantes en la estructura del hábitat, principalmente en el río Infiernillo, el Caño Crucitas y la quebrada Tamagá. De acuerdo a Matthews (1998) estas diferencias, desde el tipo de topografía del sistema, hasta variaciones locales específicas, influencian los patrones de distribución de las comunidades de peces tropicales. El río Infiernillo se encuentra en un área fragmentada de bosques, cuya deforestación ribereña ha contribuido al aumento en la erosión y sedimentación de los suelos (M. Espinoza obser. pers.). La variabilidad en la estructura del hábitat se debe principalmente al tipo de sustrato, el ancho del caudal y la elevación. Los fondos de limo y arcilla constituyen un alto porcentaje del sustrato en el río Infiernillo, la Quebrada Minas y el Caño Crucitas. Además, estos sitios presentan una mayor profundidad y ancho, favoreciendo la formación de pozas. En el resto de los sitios dominan los rápidos y sustratos duros. Sin embargo, en algunas quebradas como Descubrimiento y la Cascada también es común encontrar pozas de menor tamaño. La mayoría de las especies registradas durante los sondeos ecológicos fueron capturadas en pozas y no en rápidos. De acuerdo a Mota y Cox (2003) la interacción entre rápidos/pozas y los factores físicos propios de las quebradas, influyen directamente en la riqueza, composición y distribución de especies. La mayor riqueza de especies en pozas puede estar relacionada con la preferencia por ambientes profundos y anchos, con menor velocidad de corriente (Bart 1989). Schlosser (1982) observó que hábitats profundos proporcionan mayor estabilidad que los rápidos, debido a que las pozas atenúan los cambios a corta escala en velocidad de corriente, profundidad y diferencias en el sustrato. Sin embargo, la ictiofauna que habita las pozas experimenta un mayor riesgo de depredación, por lo que algunas de especies de sardinas (familia Characidae) se refugian en rápidos para evitar ser depredadas (Harvey y Stewart 1991). Es posible que otros factores, bióticos y/o abióticos, que no fueron tomados en cuenta durante los sondeos ecológicos influyan en la estructura, composición y distribución de las comunidades estudiadas (Chesson y Huntly 1997).

Estructura trófica: Durante los SER se observó que la estructura trófica de las comunidades de peces tropicales fue dominada por especies omnívoras e insectívoras. La abundancia de insectos terrestres y acuáticos en los ríos y quebradas, además de la cobertura boscosa ribereña (e.g. frutos, semillas, hojas, flores), proporcionan una gran variedad de recursos disponibles (Bussing 1998). Otras especies se alimentan de detritus, algas, escamas de peces $\mathrm{u}$ otros invertebrados. A pesar del alto número de insectívoros, pocas especies se especializan exclusivamente en una sola fuente de alimento, lo cual explica el alto número de especies omnívoras (Ortaz 1992, Rojas 2002). Greenberg (1991) encontró que es común que exista una repartición adecuada de recursos en especies similares, que utilizan una misma fuente de 
alimento. Sin embargo, la competencia interespecífica por un recurso también puede llevar a una segregación espacial, en donde las especies no sólo difieren en el tipo y calidad de su dieta, sino también en los períodos de actividad en los que se alimentan, evitando así la competencia por un mismo recurso. Además Mota y Cox (2003) sugieren que la segregación espacial también puede estar asociada con el tamaño o la disponibilidad de micro-hábitats, como se ha discutido anteriormente. Harvey y Stewart (1991) encontraron que peces de mayor tamaño frecuentemente se encuentran en hábitats profundos. Los individuos más grandes fueron observados en el río Infernillo, en donde existe una gran cantidad de hábitats profundos. Algunas especies de sardinas de mayor tamaño como la machaca (B. guatemalensis), los guapotes (Parachromis spp.) y el pez gaspar (A. tropicus) son comunes en el río infiernillo. Estas especies son principalmente piscívoras, y es posible que tengan un efecto importante en la distribución de otras especies de menor tamaño, limitando su acceso a recursos (alimento y espacio) para el crecimiento y reproducción (Esteves y Galetti 1995, Arahna et al. 1998).

Consideraciones ecológicas: A través de los estudios que se han realizado en el área de la minería de oro en el Cerro Crucitas (SER) se ha generado una fuente de información importante para evaluar el sistema biológico acuático y medir los cambios antropogénicos que puedan alterar el equilibrio de este sistema. Existe una gran riqueza de especies en los ríos y quebradas cercanas al área de perturbación, y además, la zona no ha experimentado reducciones evidentes o desapariciones locales a través de los años. Comunicaciones personales aportan información de la presencia de otras especies de gran importancia biológica como el pez sierra (Pristis pectinata) y el gaspar $A$. tropicus, que son especies de gran fragilidad, debido al estado crítico de sus poblaciones, producto de la intensa actividad pesquera y la pérdida o fragmentación de hábitats acuáticos, principalmente humedales y lagunas naturales
(Campos y Umaña 1986, Adams et al. 2006). Además, el pez sierra pertenece a la lista roja de especies en peligro de extinción (Adams et al. 2006), por lo tanto, esta y otras especies vulnerables o en riesgo de extinción, representan elementos fundamentales en la conservación de los ambientes acuáticos de la región norte de Costa Rica.

Las actividades de extracción de la saprolita en el Cerro Crucitas no han iniciado propiamente, y aunque es difícil predecir los impactos de una minería de oro sobre el ambiente, es muy posible que las consecuencias inmediatas sean: 1) cambios en el flujo del caudal, 2) aumento en la descarga de sedimentos y nutrientes al sistema, y 3) contaminación química por escorrentías hacia los ríos. De acuerdo a Rojas (2002), la construcción de los diques afectará el caudal de Minas, que será desviado hacia otra cuenca, por lo tanto Minas podría desaparecer o reducirse notablemente en épocas del año con menor precipitación. El movimiento de tierras, construcción de diques y relaves, y la fuerte precipitación que afectan mayormente a los suelos arcillosos de alta porosidad, pueden producir un aumento en la descarga de sedimentos hacia los ríos y quebradas (Solís-Porras 1993, Ngcobo 2006). Este impacto modificará el tipo de sustrato (fragmentación y deterioro del hábitat), la cantidad de materia orgánica presente (eutroficación del sistema) y la visibilidad en el medio acuático (reducción de la producción primaria), produciendo dos tipos de cambios inmediatos: 1) cambios en la estructura trófica, abundancia y distribución de especies, y 2) cambios en la salud del sistema, que pueden traducirse en un aumento en la mortalidad de peces. Otro impacto importante y difícil de cuantificar, en sus etapas iníciales, es la introducción de residuos químicos al sistema (Ramírez et al. 1985, Howarth 2000). En varias minerías de oro en Brasil, se han reportado altas concentraciones de mercurio y cianuro de sodio en los ríos y quebrada, acumulándose en los tejidos de peces, lo cual puede tener un efecto negativo sobre niveles tróficos superiores debido a los mecanismos de bio-acumulación de contaminantes (Colombo et al. 2000). 
Cualquier actividad antropogénica puede generar cambios negativos sobre los ambientes acuáticos, sin embargo, el impacto podría reducirse notablemente con la implementación de medidas que minimicen los riesgos, como el mejoramiento en los estándares de técnicas de extracción, protocolos y planes de contingencia, el control de las tasas de sedimentación y la reforestación ribereña. Además, la prevención ante posibles derrames de residuos químicos podría mitigar los efectos negativos de la actividad sobre los sistemas acuáticos. Finalmente, los monitoreos biológicos y químicos deben ser fundamentales en el desarrollo de actividades de extracción a gran escala. En este estudio se encontró que la heterogeneidad espacial cumple un rol importante en la dinámica de las comunidades de peces tropicales. El deterioro de los ambientes acuáticos, o simplemente la fragmentación de los sistemas naturales, pueden afectar de forma directa la composición, estructura y distribución de especies. Un mejor entendimiento del uso de hábitats específicos, así como la identificación de hábitats esenciales (e.g. sitios de reproducción, zonas de crianza y áreas de reclutamiento) pueden mejorar el desarrollo de estrategias de manejo y conservación de peces tropicales, aumentando la diversidad local y por ende la importancia biológica de la zona.

\section{AGRADECIMIENTOS}

Agradezco a Rebeca Quezada Céspedes de la Universidad Nacional por su extensa colaboración durante los muestreos de campo, la identificación y el procesamiento de los especímenes, a Esteban Bermúdez y a la consultora ambiental DEPPAT por el apoyo logístico en el planeamiento de las giras y trabajo de campo, a Sandra Arredondo de Industrias Infinito por su interés y colaboración durante la realización del presente informe y los estudios previos realizados en la zona del proyecto minero, y finalmente al Centro de Investigación Marina y Limnología (CIMAR) y Museo de Zoología de la Universidad de Costa Rica por el préstamo del equipo de pesca y el uso de las instalaciones para el análisis de datos.

\section{RESUMEN}

El impacto de las minerías de oro ha generado una gran preocupación por el deterioro de los hábitats acuáticos y la fragmentación de los sistemas naturales. Las perturbaciones antropogénicas sobre la estructura y heterogeneidad del sistema pueden influir en la estabilidad de las comunidades acuáticas. Sondeos ecológicos rápidos (1996, 2002, 2007) fueron empleados para determinar la estructura, composición y distribución de las comunidades de peces tropicales en varios ríos y quebradas del área de una minería de oro en el Cerro Crucitas, Costa Rica. Además, la composición de especies y abundancia relativa se relacionó con la estructura del hábitat. Se registró un total de 35 especies, de las cuales la sardina Astyanax aeneus (Characidae) y la olomina Alfaro cultratus (Poeciliidae) fueron las especies más abundantes (71\%). La mayor riqueza de especies se observó en el Caño Crucitas ( $\mathrm{s}=$ 19) y la quebrada Minas $(\mathrm{s}=18)$. Se encontró una gran variación en la estructura y composición de las comunidades de peces, principalmente, en el río Infiernillo y Minas $\left(\lambda=0.0, \mathrm{~F}_{132,66}=2.24, \mathrm{p}<0.001\right)$. La presencia o ausencia de algunas especies como Gobiomorus dormitor, Rhamdia nicaraguensis, Parachromis loiseillei y Atractosteus tropicus explicaron la mayor parte de la variación espacial entre sitios. La heterogeneidad estructural del hábitat también contribuyó a explicar diferencias importantes entre sitios $\left(\lambda=0.004, \mathrm{~F}_{60,183}=5.52, \mathrm{p}<0.001\right)$. El tipo de sustrato (blando y duro) y la estructura del hábitat (elevación, ancho y profundidad) explicaron la mayor parte de la variabilidad observada en el río Infiernillo, el Caño Crucitas y la quebrada Tamagá. Además se encontró una asociación significativa entre las especies y la estructura del hábitat. Este estudio evidencia la complejidad en la estructura y composición de especies que habitan el área de la minería de oro, y acentúa la importancia de la heterogeneidad estructural del hábitat en la dinámica de las comunidades de peces tropicales. El deterioro y fragmentación de los hábitats acuáticos en el área de una minería de oro pueden tener un fuerte efecto negativo sobre la estructura de las comunidades de peces tropicales.

Palabras claves: comunidades de peces tropicales, disturbios antropogénicos, minería de oro, Costa Rica, sondeos ecológicos rápidos, composición de especies.

\section{REFERENCIAS}

Akçay, M. \& C.J. Moon. 2004. The environmental impact of mining in the Pontides, Turkey: reconnaissance sampling and GIS-based analysis. Geochem. Exp. Environ. Anal. 4: 317-328.

Arahna, J.M., D.F. Takeuti \& T.M. Yoshimura. 1998. Habitat use and food partitioning of fishes in a coastal stream of Atlantic Forest, Brazil. Rev. Biol. Trop. 46: 951-959. 
Ault, T.R. \& C.R. Johnson. 1998. Spatial variation in fish species richness on coral reefs: habitat fragmentation and stochastic structuring processes. Oikos 82: 354364.

Bart, J.R. 1989. Fish habitat association in Ozark stream. Env. Biol. Fish. 24: 173-186.

Burcham, J.K. 1988. Fish communities and environmental characteristics of two lowland streams in Costa Rica. Rev. Biol. Trop. 36: 273-285.

Bussing, W.A. 1976. Geographic distribution of the San Juan Ichthyofauna of Central America with remarks on its origin and ecology, p. 157-175. In T. B. Thorson (ed.). Investigations of the Ichthyofauna of Nicaraguan Lakes. University of Lincoln, Nebraska, EEUU.

Bussing, W.A. 1993. Fish communities and environmental characteristics of a tropical rain forest river in Costa Rica. Rev. Biol. Trop. 41: 791-809.

Bussing, W.A. 1994. Ecological aspects of the fish community, p. 195-198. In L. McDade, K.S. Bawa, H.A Hespenheide \& G.S. Hartshon (eds.). La Selva, ecology and natural history of a tropical rain forest. University of Chicago, Chicago, EEUU.

Bussing, W.A. 1998. Peces de las aguas continentales de Costa Rica. $2^{\text {da }}$. Ed. Editorial Universidad de Costa Rica. San José, Costa Rica.

Bussing, W.A. \& M.I López. 1977. Distribución y aspectos ecológicos de los peces de las cuencas hidrográficas de Arenal, Bebedero y Tempisque, Costa Rica. Rev. Biol. Trop. 25: 13-37.

Campos, J.A. \& G. Umaña. 1986. Las lagunas de inundación del Refugio Nacional de Vida Silvestre Caño Negro. Biocenosis 3: 24-26.

Castilhos, Z.C., E.D. Bidone \& L.D. Lacerda. 1998. Increase of the background human exposure to mercury through fish consumption due to gold mining at the Tapajós River Region, Pará State, Amazon. Bull. Environ. Cont. Tox. 61: 202-209.

Chesson, P.L. \& Huntly, N. 1997. The roles of harsh and fluctuating conditions in the dynamics of ecological communities. Am. Nat. 150: 520-553.

Colombo, J.C., C. Bilbos, M. Remes, D. Colautti, P. Landoni \& C. Brochu. 2000. Detritivorous fish contamination in the Río de la Plata estuary: a critical accumulation pathway in the cycle of anthropogenic compounds. Can. J. Fish. Aquat. Sci. 57: 1139-1150.
Digby, P.G.N. \& R.A. Kempton. 1987. Multivariate Analysis of Ecological Communities. Chapman \& Hill, Londres, Reino Unido.

Espinoza, M. \& E. Salas. 2005. Estructura de las comunidades de peces de arrecife en las Islas Catalina y Playa Ocotal, Pacífico Norte de Costa Rica. Rev. Biol. Trop. 53: 523-536.

Esteves, K.E. \& P.M. Galetti. 1995. Food partitioning among some characids of a small Brazilian floodplain lake from the Paraná River basin. Env. Biol. Fish. 42: $375-389$.

Freitas, C.E., M. Petrere \& W. Barbella. 2005. Natural and artificially-induced habitat complexity and fresh water fish species composition. Fish. Manag. Ecol. 12: 63-67.

Gotelli, N.J., \& A.M. Ellison. 2004. A primer of Ecological Statistics. Sinauer Assoc. Inc. Massachusetts, Boston, EEUU.

Guilliam, J.F., D.F. Fraser, M. \& Alkins-Koo. 1993. Structure of tropical stream fish community: a role for biotic interactions. Ecology 74: 1856-1870.

Greenberg, L.A. 1991. Habitat use and feeding behavior of thirteen species of benthic stream fishes. Environ. Biol. Fish. 31: 389-401.

Harvey, B.C. \& A.J. Stewart. 1991. Fish size and habitat depth relationships in headwater streams. Oecologia 87: 336-342.

Honran, D.L., J.L Kershner, C.P Hawkings \& T.A. Crowl. 2000. Effects of habitat area and complexity on Colorado River cutthroat trout density in Uinta Mountain Stream. Trans. Am. Fish. Soc. 129: 1250-1263.

Howarth, R., D. Anderson, J. Cloern, C. Elfring, C. Hopkinson, B. Lapointe, T. Malone, N. Marcus, K. MacGlathery, A. Sharpley \& D. Walker. 2000. Nutrient pollution of coastal rivers, bays, and seas. Issues in Ecol. 7: 2-15.

Ibarra, A.A., Y.S. Park, S. Brosse, Y. Reyjol, P. Lim \& S. Lek. 2005. Nested patterns of spatial diversity revealed for fish assemblages in a west European river. Ecol. Fresh. Fish. 14: 233-242.

Koenig, K.W., R.J. Beatty \& S. Martínez. 1976. Geographic distribution of the San Juan Ichthyofauna of Central America with remarks on its origin and ecology, p. 157-175. In T. B. Thorson (ed.). Investigations of the Ichthyofauna of Nicaraguan Lakes. University of Lincoln, Nebraska, EEUU. 
Konings, A. 1989. Cichlids of Central America. T.F.H. Publications, Nueva Jersey, EEUU.

Krebs, C.J. 1999. Ecological Methodology. $2^{\text {da }}$ Ed. Benjamin/Cummings Imprint, California, EEUU.

Lake, P.S. 2000. Disturbance, patchiness, and diversity in streams. J. N. Am. Benthol. Soc. 19: 573-592.

Lowe-McConnell, R.H. 1987. Ecological studies in tropical fish communities. Cambridge Univ., Cambridge, Reino Unido.

Malm, O. 1998. Gold mining as a source of mercury exposure in the Brazilian Amazon. Env. Res. 77: 73-78.

Matthews, W.J. 1986. Fish faunal "breaks" and stream order in the eastern and central United States. Environ. Biol. Fishes. 17: 81-92.

Matthews, W.J. 1998. Patterns in freshwater fish ecology. Chapman and Hall, New York. (Second printing by Kluwer Academic), EEUU.

McElligott, P. \& T. Slaney. 1996. Cerro Crucitas Project. Baseline description of aquatic habitats and fish communities: July 199 Survey. Aquatic Resource Ltd. Vancouver, B.C., Canadá (mimeo).

Meck, M., D. Love \& B. Mapani. 2006. Zimbabwean mine dumps and their impacts on river water quality - a reconnaissance study. Phy. Chem. Earth. 31:797-803.

Mota, C. \& C. Cox. 2003. Structure of fish assemblages in Amazonian streams: effects of habitats and locality. Copeia: 2003:255-262.

Ngcobo, T.A. 2006. The risks associated with mines in dolomitic compartments. J. South. African. Inst. of Min. Met. 106: 251-264.

Ormond, R.F.G. \& C.M. Roberts. 1997. Biodiversity of coral reef fish, p. 216-257. In. R.F.G. Ormond, J.D. Gage \& M.V. Angel (eds). Marine Biodiversity: Patterns and Processes. Cambridge University, Cambridge, Reino Unido.

Ortaz, M. 1992. Hábitos alimenticios de los peces de montaña neotropical. Biotropica 24: 550-559.

Porcella, D.B., C. Ramel \& A. Jernelov. 1997. Global mercury pollution and the role of gold mining: an overview. Water, Air \& Soil Poll. 97: 205-207.
Peplow, D., \& R. Edmonds. 2005. The effects of mine waste contamination at multiple levels of biological organization. Ecol. Engin. 24: 101-119.

PNUMA-OEA. 1997. Manejo ambiental y desarrollo sostenible de la Cuenca de Río San Juan. Estudio de diagnóstico de la cuenca Río San Juan y lineamientos del plan de acción. Unidad de Desarrollo Sostenible y Ambiente, Secretaría General de la OEA. Washington, D.C., EEUU.

Quinn, G.P., \& M.J. Keough. 2003. Experimental Design and Data Analysis for Biologist. Cambridge University, Cambridge, Reino Unido.

Ramírez, J.M., M.A. Sequeira \& B.C. Solano. 1985. Estudio sobre el contenido de metales pesados en los ríos del área metropolitana. Ing. Cienc. Quim. 9:11-13

Rojas, R.E. 2002. Estudio de base para la evaluación del impacto ambiental de un proyecto de minería de oro en Cerro Crucitas: descripción de la ictiofauna. Informe final para DEPPAT S.A. (mimeo), San José, Costa Rica.

Roldán, G. 1992. Fundamentos de limnología neotropical. Ed. Univ. Antioquia, Medellín, Colombia.

Solís-Porras, R.E. 1993. Determinación de la erosión hídrica y pérdida de nutrimentos en cítricos y pastos en la Región Huetar Norte (San Carlos). Tesis. Lic. en Ingeniería Agronómica. ITCR, San Carlos, Alajuela, Costa Rica.

Schlosser, I.J. 1982. Fish community structure and function along two habitat gradients in a headwater stream. Ecol. Monogr. 52: 395-414.

Venter, A. \& J.H. VanVuren. 1997. Effect of gold-mine related operations on the physical and chemical characteristics of sediment texture. Water SA 23: 249-256.

Walter, R.P. \& J.M. Haynes. 2006. Fish and coral community structure are related on shallow water patch reefs near San Salvador, Bahamas. Bull. Mar. Sci. 79: 365-374.

Wood, P.J. 1997. Biological effects of fine sediments in the lotic environment. Env. Manag. 21: 203-217.

Zar, J.H. 1999. Biostatistical Analysis. Prentice Hall $-4^{\text {th }}$ ed., Nueva Jersey, EEUU. 
Zwane, N., D. Love, Z. Hoko \& D. Shoko. 2006. Managing the impacts of gold panning activities within the context of integrated water resources management planning in the Lower Manyame Sub-Catchment, Zambezi Basin, Zimbabwe. Phy. Chem. Earth. 31: 848-856.

\section{REFERENCIA DE INTERNET}

Adams, W.F., S.L. Fowler, P. Charvet-Almeida, V. Faria, J. Soto \& M. Furtado. 2006. Pristis pectinata. In: IUCN 2007. 2007 IUCN Red List of Threatened Species. $<$ www.iucnredlist.org > . Downloaded on 25 May 2008. 